\title{
Huelga de hambre, alimentación forzada y responsabilidad profesional: alcance y consideraciones éticas
}

\author{
Hunger strike, forced-feeding and professional responsibility: \\ A scoping review of ethical considerations \\ Greve de fome, alimentação forçada e responsabilidade profissional: \\ alcance e considerações éticas
}

Francisca Reyes-Arellano' ${ }^{1 *}$, Josefina Razazi Fuenzalida ${ }^{1,2}$

Recibido: 19 de julio de 2021. Aceptado para publicación: 9 de agosto de 2021. https://doi.org/10.35454/rncm.v4supl1.331

\section{Resumen}

El problema de la huelga de hambre no es nada sencillo, y se ha discutido ampliamente desde las más diversas tradiciones filosóficas. Esta manifestación, como forma de protesta, requiere un análisis interdisciplinar que integre aspectos éticos, médicos y jurídicos. La huelga de hambre implica la decisión voluntaria de privarse de alimentos para obtener el reconocimiento efectivo de un derecho no admitido o vulnerado. Este mecanismo se utiliza como medio de presión, mediante una amplia publicidad del acto del huelguista. Una discusión moral relevante se plantea ante la cuestión de la legitimidad de administrar alimentos en contra de la voluntad del huelguista, cuando su estado de salud está gravemente deteriorado por el ayuno prolongado. Teniendo en cuenta las consecuencias fisiológicas y neurológicas que ello conlleva, cabe preguntarse razonablemente si existe verdadera libertad y competencia cuando el huelguista manifiesta el rechazo a cualquier tratamiento médico $y$, en consecuencia, persiste en la continuación de la huelga, hasta llegar a la muerte, si es necesario. El presente trabajo pretende analizar la legitimidad de la alimentación forzada, teniendo en cuenta los efectos fisiológicos y neurológicos que provoca el ayuno prolongado, que podrían tener un efecto determinante en
\end{abstract}

\section{Summary}

The problem of hunger strike is far from simple and has been widely discussed from the most diverse philosophical traditions. This manifestation as a form of protest requires an interdisciplinary analysis comprising ethical, medical and legal considerations. Hunger strike implies a voluntary decision to deprive oneself of food in order to obtain the effective recognition of a right that has not been admitted or has been violated. It is used as a means of pressure, through wide publicity of the striker's act. A relevant moral discussion arises when faced with the question of the legitimacy of administering food against the striker's will, when health is seriously deteriorated by prolonged fasting. Considering the resulting physiological and neurological consequences, we can reasonably ask ourselves whether the striker is truly free and competent to refuse medical treatment and continue to strike to the point of death if necessary. This paper aims to analyze the legitimacy of forced feeding, taking into account the physiological and neurological effects caused by prolonged fasting, which could have a determining effect on the voluntariness of the striker at the time of refusing nutrition. It should be specified that the purpose is not to question the motives leading to a hunger strike or the striker's ori-

\section{Resumo}

O problema da greve de fome não é nada fácil e tem sido amplamente discutido nas mais diversas tradições filosóficas. Essa manifestação como forma de protesto requer uma análise interdisciplinar que integre aspectos éticos, médicos e jurídicos. A greve de fome implica a decisão voluntária de privar-se de alimentos para obter o reconhecimento efetivo de um direito não admitido ou violado. Esse mecanismo é utilizado como meio de pressão, através de ampla divulgação do ato do grevista. Uma discussão moral relevante surge da questão da legitimidade de administrar alimentos contra a vontade do grevista, quando seu estado de saúde está seriamente deteriorado pelo jejum prolongado. Levando em consideração as consequências fisiológicas e neurológicas que isso acarreta, é razoável indagar se existe verdadeira liberdade e competência quando o grevista expressa a rejeição de qualquer tratamento médico e, por isso, persiste em continuar a greve até a morte se necessário. $O$ presente trabalho tem como objetivo analisar a legitimidade da alimentação forçada, levando em consideração os efeitos fisiológicos e neurológicos causados pelo jejum prolongado, que poderiam ter um efeito determinante na disposição do grevista na hora de rejeitar a alimentação. Ressalte-se que não 
la voluntad del huelguista a la hora de rechazar la alimentación. Conviene precisar que no se trata de cuestionar los motivos que inducen a la adopción de una huelga de hambre, ni la autonomía que el huelguista pudiera tener en un principio, sino sobre las decisiones adoptadas cuando ya es posible evidenciar los efectos físicos y psicológicos del ayuno.

Palabras clave: huelga de hambre, alimentación forzada, bioética, suicidio. ginal autonomy, but rather the decisions taken when there is already evidence of the physical and psychological effects of fasting.

Keywords: Hunger strike; Force feeding; Bioethics; Suicide. se trata de questionar os motivos que induzem à adoção da greve de fome ou da autonomia que o grevista pode ter em um primeiro momento, mas das decisões tomadas quando já é possível demonstrar os efeitos físicos e psicológicos do jejum.

Palavras-chave: greve de fome, alimentação forçada, bioética, suicídio.
Universidad de los Andes, Chile.

2 Hospital Metropolitano, Chile.
*Correspondencia: Francisca Reyes-Arellano. fireyes@miuandes.cl

\section{INTRODUCCIÓN}

El problema de la huelga de hambre no es nuevo, ni mucho menos pacífico; muy por el contrario, se trata de un medio de manifestación, principalmente de carácter político, ampliamente utilizado como mecanismo de protesta frente a situaciones que se califican bajo el carácter de injustas o desproporcionadas, cuyo juicio de valoración moral y jurídica está lejos de ser unívoco.

De este modo, innumerables son los casos de huelguistas de hambre que se presentan ante la opinión pública como portadores de una grandeza «que destaca en una época como la nuestra, que parece poco dada a heroísmos ${ }^{(1)}$. Sin necesidad de ahondar demasiado, la actual situación de pandemia trajo consigo diversas manifestaciones de este carácter, tal como la llevada a cabo por médicos peruanos en enero de 2021, quienes iniciaron una huelga de hambre para solicitar un mejor manejo de los recursos invertidos en el sector sanitario frente al exponencial aumento de casos producidos por la segunda ola de contagios por la enfermedad por coronavirus de 2019 (COVID-19) $^{(2)}$. Casos como el anterior se replican con diferentes protagonistas, en diferentes países y esgrimiendo los más diversos argumentos para justificar sus actos.

Lo más llamativo de este mecanismo de manifestación es la novedosa inversión producida en la lógica habitual de las agresiones a las que estamos acostumbrados. En este caso, el daño de la agresión no es sufrido por el oponente (como habríamos de suponer), sino por el mismo manifestante, quien lleva a cabo la huelga de hambre. Entonces, la huelga de hambre «se convirtió en un medio para demostrar la propia superioridad moral sobre el enemigo, representado en este caso por el Estado» ${ }^{(3)}$.

Por su parte, las aristas relacionadas con la atención sanitaria de personas en huelga de hambre suscitan las más diversas opiniones y controversias en torno a los conflictos de valores que estas pueden despertar en los médicos, llamados al tratamiento de las complicaciones derivadas de la manifestación. En este sentido, la alimentación forzada se presenta como el principal dilema ético en relación con la tensión de los bienes en conflicto (vida, libertad y autodeterminación), pues la persistencia del ayuno implica riesgos ciertos para la vida y la salud de los individuos ${ }^{(1)}$, situación ante la cual el médico se ve enfrentado a la decisión de restablecer la salud por todos los medios disponibles (incluida la alimentación forzada) u omitir cualquier clase de acción que involucre atentar contra la manifestación de voluntad del huelguista.

Ahora bien, aunque en principio nos parece del todo razonable que el médico debe respetar la voluntad del huelguista que manifiesta su rechazo frente a un tratamiento, tal como se sigue de la lógica tras el rechazo de la alimentación e hidratación emprendida por un paciente consciente hospitalizado ${ }^{(4)}$, consideramos que el caso en cuestión requiere de precisiones todavía más acuciosas en lo que respecta a la competencia para rechazar la alimentación forzada, pues tal como analizaremos a continuación, aunque al comenzar la huelga de hambre existe plena consciencia y, por tanto, una voluntad verdaderamente libre e informada para consentir, el inevitable progreso del ayuno conllevará consecuencias fisiológicas que impactarán directamente en las debidas condiciones que un paciente debe cumplir 
para el rechazo del tratamiento, lo que puede llevarnos a la pregunta acerca de si el huelguista de hambre es verdaderamente competente para rechazar un tratamiento médico, tal como la realimentación.

\section{ALCANCE Y DELIMITACIÓN DE LOS CONCEPTOS A UTILIZAR: HUELGA DE HAMBRE Y ALIMENTACIÓN FORZADA}

\section{Huelga de hambre}

Dada la álgida discusión doctrinal, jurídica y filosófica en torno a este concepto, no existe una definición unívoca con respecto a lo que se considera como una huelga de hambre para efectos jurídicos, éticos o médicos. No obstante, entre los diferentes esfuerzos por otorgar una definición, es posible vislumbrar algunos elementos comunes que subyacen y caracterizan a lo que habitualmente se considera como una huelga de hambre para todos los efectos prácticos.

Por una parte, una definición sistemática, proveniente desde lo que podría considerarse como la tradición clásica de la ética, considera a esta conducta como la «abstención total de alimento, emprendida para obtener la satisfacción de determinadas reclamaciones, con la decisión de llevarla hasta la muerte si no se da satisfacción a las reivindicaciones que la motivan ${ }^{(5)}$. Por otra parte, desde una dimensión más bien jurídicosocial, la huelga de hambre se califica como «una forma de protesta social pacífica y extrema al mismo tiempo, en la que se tensionan valores centrales del individuo, como la vida y la libertad» ${ }^{(6)}$.

A su vez, el Departamento de Ética del Colegio Médico de Chile, tomando lo estipulado por los abogados Precht y Faundes, ha definido a la huelga de hambre como «la abstinencia voluntaria de ingesta de alimentos o líquidos por un período de tiempo definido o no, utilizada como una herramienta de denuncia y protesta para reivindicar el cumplimiento de algún derecho, eliminar reglas o normas consideradas ilegítimas o injustas por el sujeto que la realiza, pretendiendo así modificar una determinada disposición de la autoridad, un grupo o la comunidad, arriesgando con ello la propia vida $y$ salud» ${ }^{(6,7)}$.

En un contexto médico, una huelga de hambre se define como la negativa de ingesta de alimentos usada como forma de protesta o demanda ${ }^{(8)}$. Mientras que la Declaración de Malta lo define como el rechazo a la ingesta por un «período significativo», sin definir con claridad cuánto tiempo es ${ }^{(9)}$. La mayoría de las recomendaciones coinciden en que se debe realizar una valora- ción clínica a las 72 horas de comenzado el ayuno ${ }^{(10)}$. Con respecto al tiempo o impacto significativo, hay autores que señalan que una pérdida de aproximadamente el $18 \%$ del peso corporal resulta en problemas médicos serios, mientras que una pérdida del $30 \%$ implica una amenaza vital ${ }^{(10)}$. Cabe considerar también que la alimentación forzada de una persona desnutrida puede llevar a síndrome de realimentación, cuadro clínico de riesgo vital, por lo que esta se debe realizar con particular cuidado en este grupo de personas.

Ahora bien, no existe un límite claro sobre el momento en el que comienzan a ocurrir los cambios fisiológicos, neurológicos y psicológicos propios del ayuno. Tal como se puede vislumbrar, aunque cada definición difiere de la anterior, es posible encontrar en ellas un conjunto de características que podríamos considerar como comunes a toda huelga de hambre. Estas son:

- la decisión voluntaria de privarse de alimento;

- la finalidad de conseguir el reconocimiento efectivo de un derecho juzgado, conculcado o no admitido;

- el procedimiento de presión sobre una persona o una institución causante de la injusticia reclamada;

- la táctica de dar la máxima publicidad posible a la acción huelguista $^{(6)}$.

Todo lo anterior constituye a la huelga de hambre en una acción especialmente compleja de evaluarse desde el punto de vista moral, pues varias serán las consideraciones éticas que se deberán tener en cuenta para calificar su legitimidad o ilegitimidad, tales como la finalidad del sujeto que actúa, la posibilidad (directa o indirecta) de llegar a morir o ver afectada su salud o la eventual calificación de su acción como un suicidio condicional.

En cualquier caso, el estudio de la huelga de hambre $\ll$ no puede separarse del contexto ni de las causas o motivaciones de quienes la sostienen ${ }^{(6)}$, pues, como señalamos anteriormente, se trata de una herramienta utilizada como mecanismo de presión para visibilizar ciertas demandas sociales que no han sido oídas, o solo se han atendido de manera parcial.

\section{Alimentación forzada}

No existe una definición ampliamente aceptada o sistemática sobre la alimentación forzada en el contexto de huelga de hambre. Ahora bien, este parece ser un concepto tautológico: es una alimentación que se realiza de manera forzosa; es decir, sin el consentimiento de quien es alimentado. 
Cabe hacer la distinción entre diferentes alternativas para realizar este procedimiento ${ }^{(11)}$, y la alimentación por medio de la sonda nasogástrica u orogástrica es la más ampliamente utilizada. Sin embargo, también existe la posibilidad de administrar alimentación por vía intravascular, método que adquiere especial relevancia en aquellos casos en los que la persona lleva más tiempo sin alimentación, por lo que se hace importante realizar correcciones electrolíticas y administración de macronutrientes en dosis crecientes, iniciando con $10 \mathrm{kcal} / \mathrm{kg} /$ día para prevenir el síndrome de realimentación ${ }^{(12,13)}$.

\section{CONSIDERACIONES ÉTICAS}

\section{Huelga de hambre y suicidio}

La primera consideración ética relativa a la huelga de hambre dice relación con dilucidar lo que varios autores consideran que podría calificarse como una acción «condicionalmente suicida». A este respecto pueden seguirse dos líneas argumentativas completamente contrapuestas: aquella que une la huelga de hambre y la conducta suicida (como un ilícito o conducta moralmente reprochable), y aquella que estima que no puede calificarse a la huelga de hambre como una conducta suicida por tratarse de dos acciones caracterizadas de manera diferente y, por ende, cuya valoración moral es también diversa.

Podemos preguntarnos si resultan aplicables las mismas razones que permitirían calificar al suicidio como un acto moralmente reprochable para el caso de la huelga de hambre. En este sentido, se podría afirmar que «si la intención del huelguista es seria, la propuesta que adopta incluye, como uno de sus fines próximos, la destrucción de su propia vida ${ }^{(1)}$. Haciendo una lectura de base kantiana, quien se somete a una huelga de hambre con la finalidad de ejercer presión para modificar una decisión política, no haría otra cosa que disponer de su vida como un medio para otra cosa. De esta forma, «disponerse como un mero medio significa degradar a la humanidad en su persona ${ }^{(14)}$. Se trataría entonces de una instrumentalización de la vida. De la misma manera, en sus «Lecciones de ética», Kant consagra que «quien contraviene los deberes para consigo mismo tiene en poco a la humanidad y no queda en situación de poner en práctica los deberes para con los demás ${ }^{(15)}$.

Ahora bien, volviendo a la discusión sobre la moralidad del suicidio, se debiese advertir que el derecho a quitarse la vida «entra en franca colisión con el deber general y primario de respetar la vida propia» ${ }^{(16)}$.
Entonces, quien comete suicidio, aun cuando este se realice de manera lenta, progresiva y condicional, como sería el caso de la huelga de hambre, sobrepasará «todos los límites del uso 'legítimo' de su libre arbitrio» ${ }^{(15)}$. Para comprender mejor el problema de la intención condicional del huelguista de hambre se sugiere revisar a Miranda y colaboradores ${ }^{(1)}$.

En el sentido contrario, quienes argumentan en la línea de considerar a la huelga de hambre como una acción no suicida sostienen que lo buscado por el huelguista no es una acción mala en sí; por el contrario, existiría una razón justa y proporcionada como motivo para actuar $^{(6)}$. La muerte, «a pesar de ser una consecuencia posible del ayuno prolongado, no es buscada como un fin en sí misma, sino que es el riesgo que libremente las personas en huelga de hambre asumen en pos del objetivo que han definido como prioritario» ${ }^{(6,7)}$.

De alguna manera, esta forma de argumentar podría enmarcarse dentro del principio de razonamiento práctico conocido como «doble efecto», el cual busca dilucidar las condiciones que deben concurrir para evaluar la licitud de una acción que «produce o puede producir dos efectos, de los cuales uno es bueno y el otro es malo» ${ }^{(17)}$. Lo característico de este principio es que el efecto bueno sea querido por el agente, mientras que el efecto malo sea sencillamente tolerado. De este modo, todo indicaría que, al aceptar la muerte como una consecuencia posible del ayuno prolongado, con miras a la obtención de un efecto bueno constituido por la consecución del objetivo propuesto, el huelguista de hambre podría actuar en virtud de este principio.

No obstante, si se revisan con detenimiento los requisitos necesarios para que la acción de doble efecto se considere lícita, deberán concurrir simultáneamente que: (i) el efecto malo no se busque como fin ni como medio, y (ii) que exista una razón proporcionalmente grave para aceptarlo ${ }^{(17)}$. De esta forma, la bondad de la acción debe asegurarse no solo a nivel de los fines, sino de los medios, razón por la cual no debe existir relación de causalidad entre el efecto malo y el efecto bueno, lo que sí ocurriría en el caso del huelguista de hambre, pues en este caso, el efecto bueno se produciría a través de la realización del efecto malo; por ello, no resulta aplicable este principio.

Aun así, para algunos autores seguirá siendo «lógica y éticamente incorrecto tratar la huelga de hambre siempre en el contexto temático del suicidio» ${ }^{(6)}$, ya que, a su juicio, ambos constituyen realidades diametralmente distintas. 


\section{Alimentación forzada y responsabilidad profesional}

En el contexto de una huelga de hambre, especialmente si esta se extiende durante un período prolongado, pueden comenzar a suscitarse diferentes complicaciones para el estado de salud del huelguista. Las más precoces van desde trastornos metabólicos y endocrinos, dado que en un estado de ayuno persistente, al no haber ingesta de glucosa como transporte energético, el organismo torna su maquinaria metabólica hacia el glucógeno hepático, en primera instancia, para obtener glucosa por medio de la glucogenólisis y, finalmente, por medio de la neoglucogénesis, a partir del catabolismo de las proteínas estructurales. En etapas ulteriores se describen trastornos neurológicos, que causan también cambios a nivel psiquiátrico, como lo es la disminución global de la actividad serotoninérgica. Esto se ha evidenciado principalmente con la down regulation de los receptores de serotonina, sobre todo en la corteza frontal, lo cual justifica los cambios de comportamiento descritos en sujetos en ayuno prolongado, como lo son la agresividad y la impulsividad; pudiendo llegar a extremos tales como trastornos disociativos, alucinaciones y brotes de psicosis. Estos cambios a nivel de neurotransmisores se evidencian igualmente en la disminución de serotonina, al nivel del líquido cerebroespinal y en los niveles séricos de triptófano.

Dichas complicaciones hacen necesaria la intervención de profesionales de la salud, los cuales interactúan con el huelguista bajo la figura de médicos tratantes. En este contexto, uno de los principales dilemas se presenta cuando el ayuno prolongado se transforma en una amenaza para la conservación de la vida del individuo. Al respecto, es preciso señalar que en la literatura no existe claridad de qué se entenderá por «ayuno prolongado», así como tampoco del momento exacto en el cual el ayuno se constituye como una amenaza cierta para la vida del huelguista, pues todo ello dependerá, en gran medida, de las condiciones fisiológicas previas de la persona.

Frente a dicho escenario, el médico tratante debe afrontar, con especial cautela, la toma de decisiones terapéuticas, de tal manera que respete la autonomía del paciente $y$, a su vez, procure que este comprenda y pondere los riesgos de su decisión. De ninguna manera puede el profesional médico ejercer coacción o presión alguna para que el huelguista revierta su decisión, pues esto constituye un atentado directo en contra de su autodeterminación.
Con todo, las directrices para la praxis médica en el contexto de una huelga de hambre parecieran no ser del todo determinantes, pues estas quedan sujetas al análisis de una serie de variables que solo pueden ponderarse en un contexto concreto y específico. Entonces, en el caso particular de la alimentación forzada, la Declaración de Malta, adoptada por la 43. ${ }^{\text {a }}$ Asamblea Médica Mundial en noviembre de 1991, consagra entre sus principios que: "4. El médico debe respetar la autonomía de la persona. Esto puede incluir una evaluación difícil, ya que los deseos reales de la persona en huelga de hambre puede que no sean tan claros como parecen. Toda decisión pierde fuerza moral si se toma bajo amenazas, presión o coerción de los pares. No se debe obligar a las personas en huelga de hambre a ser tratadas si lo rechazan. Aplicar, dar instrucciones o ayudar a la alimentación forzada contra un rechazo informado y voluntario es injustificable. La alimentación artificial con el consentimiento explícito o necesariamente implícito de la persona en huelga de hambre es aceptable éticamente" ${ }^{(9)}$.

Por consiguiente, los profesionales sanitarios sujetos a la decisión de aplicar un plan terapéutico a la persona en huelga de hambre, especialmente si se trata de alimentación forzada, deben procurar dilucidar, en primer lugar, cuál es la voluntad real del huelguista, para luego evaluar si la manifestación de voluntad se realizó con plena competencia y voluntariedad ${ }^{(17)}$, de manera que seguir y respetar dicha manifestación se constituya como una máxima para la actuación médica.

\section{CONSIDERACIONES MÉDICAS Y FISIOLÓGICAS}

En este análisis, requiere de especial consideración el rol que desempeñan las condiciones fisiopatológicas inducidas por el ayuno prolongado. Estas pueden verse reflejadas a nivel metabólico, hematológico, musculoesquelético, endocrino e, incluso, psicológico y psiquiátrico. Cabe destacar que la literatura sobre consecuencias fisiopatológicas del ayuno en el contexto de huelga de hambre es escasa. Esto se explica por la dificultad metodológica y ética que supone el estudio y reporte de este tipo de casos. La mayoría de la literatura sobre las consecuencias fisiológicas del ayuno prolongado describe las alteraciones a nivel de neurotransmisores en modelos animales ${ }^{(18)} \mathrm{y}$ en el contexto de ayuno prolongado en personas obesas; sin embargo, muchos de los efectos descritos en ellos son extrapolables a personas que, teniendo un estado de salud previamente 
sano, deciden comenzar un ayuno prolongado en el contexto de huelga de hambre ${ }^{(7,19)}$.

A nivel neurológico, durante el ayuno prolongado se destaca la disminución de transportadores serotoninérgicos, sobre todo en la corteza frontal ${ }^{(20)}$; además de la reducción del ácido 5-hidroxindolacético (5-HIAA), metabolito principal de la degradación de la serotonina, y disminución del triptófano plasmático. Estas alteraciones reflejan una reducción global de la actividad serotoninérgica, lo que representa un fundamento fisiológico suficiente para justificar cambios de comportamiento, con rasgos de impulsividad e hiperirritabilidad $^{(18,21)}$.

También en el ámbito psiquiátrico se describen trastornos en torno al ayuno prolongado. Diversos autores han descrito la ocurrencia de somatización, disociación, confusión, hiperirritabilidad y agresividad ${ }^{(22-24)}$, así como delirios de persecución, alucinaciones visuales y auditivas e, incluso, ideación suicida ${ }^{(21)}$. Estas alteraciones también están descritas en casos emblemáticos, como el de uno de los huelguistas irlandeses en $1981^{(25)}$, que cursó con un aparente cuadro disociativo; y el brote psicótico descrito en el caso de huelga de hambre en Australia realizado por un extranjero en el contexto de asilo político en $1996^{(26,27)}$.

\section{EL PROBLEMA DE LA VOLUNTARIEDAD}

Como se mencionó antes, para que se constituya una decisión verdaderamente libre en torno al consentimiento informado, se requiere que se cumplan los elementos de competencia, voluntariedad, exposición de la información material, recomendación, comprensión, decisión y planificación ${ }^{(18)}$. Cabe destacar que para que se cumpla el elemento de competencia y voluntariedad debemos estar frente a una persona con su juicio conservado, lo que incluye un estado psicológico y neurológico sin alteraciones, y justamente este aspecto se altera, en mayor medida, por el ayuno prolongado.

A este respecto, la literatura médica parece no conciliar la evidente tensión entre las condiciones necesarias para que se exprese una voluntad verdaderamente libre e informada, y las reales condiciones de deterioro paulatino y exponencial que el huelguista sufre durante el ayuno, las cuales son reconocidas por los mismos autores. De este modo, pareciera hacerse una interpretación inapropiada del conocido principio de autonomía, esgrimiendo que cualquier acto realizado en virtud de este principio (como sería el rechazo de la alimentación, en este caso) sería necesariamente proporcionado, jus- tificado, razonable e incluso exigible; sin embargo, se deja de lado que este principio ético rector de la praxis médica siempre se ejerce en un contexto determinado y, por ello, su ejercicio no consistiría en una mera manifestación de deseos, sino que debe estar acompañado por un cierto grado de competencia y voluntariedad para entender y decidir elementos iniciales y condiciones previas para ejercer tal derecho.

Entonces, una persona puede manifestar libremente su voluntad de iniciar una huelga de hambre cuando sus condiciones fisiológicas son óptimas, o por lo menos estables. De tal modo, habrá que hacer una distinción entre esta manifestación inicial de la voluntad, de aquellas que se realicen en etapas ulteriores, cuando ya ha pasado un tiempo en ayuno; específicamente por el efecto que produce el ayuno sobre el sistema nervioso central. Esta distinción es fundamental, dado que la valoración por el profesional de la salud no ocurre previo al inicio de la huelga, cuando la persona se encuentra, en el mejor de los escenarios, en condiciones de salud óptimas, sino una vez que se evalúa la opción de forzar la alimentación. En este contexto, la negativa por parte del huelguista no se puede considerar una decisión libre, debido a las alteraciones a nivel psicológico y psiquiátrico antes mencionadas.

Entonces, aunque concordamos plenamente con que «la toma de decisiones acerca del tratamiento médico recae, por tanto, sobre el paciente, y es el paciente mismo quien, al exponer su libre elección, debe tener en cuenta los bienes en cuestión $(. ..){ }_{\gg}{ }^{(28)}$, esta toma de decisiones siempre debe realizarse bajo condiciones de plena capacidad; es decir, suponiendo la voluntariedad para entender y decidir. Entonces, «la libertad presupone, de hecho, la información y el consentimiento informado» ${ }^{(28)}$.

El Colegio Médico de Chile señala que de las decisiones médicas se deben «proponer solo las intervenciones consentidas por el individuo, respetando su rechazo a la alimentación. Para esto debe cumplirse con todas las formalidades de un rechazo válido a un tratamiento; es decir, información, voluntariedad y competencia, y esta expresión de su voluntad debe quedar consignada en la ficha clínica o en un documento específico» ${ }^{(7)}$. Como es posible vislumbrar, el rechazo de la alimentación, y la consiguiente obligación de no aplicar tratamiento por parte del personal médico, debe consagrarse como pauta de acción, considerando siempre que dicha decisión se manifestó bajo las condiciones básicas de voluntariedad y competencia. 


\section{CONCLUSIÓN}

Tal como ha sido posible constatar en el presente trabajo, resulta indiscutible que cualquier intento por aproximarse al análisis de la huelga de hambre, la alimentación forzada y el rol que el profesional médico debe adoptar frente al huelguista distará de ser sencillo, especialmente si se tratan de abordar las consideraciones éticas y fisiológicas que envuelve (con especial dramatismo) la gran mayoría de las situaciones en que se presentan dilemas de este tipo.

En general, se pueden agrupar los ejes centrales del presente artículo en torno a las siguientes ideas:

- Aunque no existe una definición unívoca de lo que se entenderá por huelga de hambre y alimentación forzada, se pueden encontrar algunos elementos comunes que permitan caracterizar e identificar a ambas clases de acciones.

- Desde la perspectiva de la evaluación moral de la acción, la huelga de hambre se puede considerar como un suicidio, en cuanto el huelguista dispone de su vida como un medio para alcanzar un objetivo ulterior, asumiendo la posibilidad de la muerte como una consecuencia previsible de la acción.

- La intervención médica del profesional tratante debe ser especialmente cautelosa en una situación de huelga de hambre, pues esta debe procurar conciliar la voluntad del huelguista, a la vez que intenta dilucidar si dicha voluntad es verdaderamente competente.

- El ayuno prolongado producirá una serie de efectos fisiológicos que tornarán cada vez más difícil la capacidad de decisión del huelguista.

- Solo se puede afirmar con certeza que el consentimiento inicial de la huelga puede ser verdaderamente libre e informado; sin embargo, las ulteriores manifestaciones de voluntad podrían verse alteradas por los trastornos fisiológicos propios del ayuno prolongado, razón por la cual no puede afirmarse con plena certeza que el huelguista consienta con plena competencia y voluntariedad, elementos mínimos para el rechazo de un tratamiento médico, como sería el caso de la alimentación forzada.

Aun así, se considera que la literatura disponible en la materia sigue siendo insuficiente frente a la gran cantidad de aristas e interrogantes del problema, que siguen quedando inconclusas. Frente a esto, el presente trabajo, aún sujeto a ciertas limitaciones, ha pretendido ser un aporte para la reflexión acerca de esta cuestión.

\section{Fuente de financiación}

El presente estudio no tuvo financiación.

\section{Conflicto de intereses}

Las autoras declaran no tener ningún conflicto de intereses.

\section{Declaración de autoría}

F. Reyes-Arellano y J. Razazi contribuyeron igualmente a la concepción y diseño de la investigación. Todos los autores revisaron el manuscrito, acuerdan ser plenamente responsables de garantizar la integridad y precisión del trabajo, y leyeron y aprobaron el manuscrito final.

\section{Referencias bibliográficas}

1. Miranda-Montecinos A, García-Huidobro J, ContrerasAguirre S. La huelga de hambre como suicidio intencional. Una propuesta de valoración moral desde la tradición Central de la ética. Pers Bioét. 2015;19(1):64-79. doi: 10.5294/ PEBI.2015.19.1.6

2. Arias T. Grupo de médicos peruanos está en huelga de hambre en medio de la creciente segunda ola de casos de covid-1 [Internet]. CCN Latinoamérica; 2021 [consultado el 16 de junio de 2021]. Disponible en: https://cnnespanol.cnn. com/2021/01/21/grupo-de-medicos-peruanos-esta-enhuelga-de-hambre-en-medio-de-la-creciente-segunda-ola-decasos-de-covid-19/

3. Riederer C. Die RAF und die Folterdebatte der 1970er Jahre. Wiesbaden: Springer VS; 2014.

4. Requena P. ¡Doctor, no haga todo lo posible! De la limitación a la prudencia terapéutica. $1^{\text {a }}$ edición. Granada: Comares S.L.; 2017.

5. Elizari FJ. Bioética. Madrid, España: Ediciones Paulinas; 1991.

6. Precht J, Faundes JJ. Legitimidad de la huelga de hambre: un debate sobre el derecho a la vida y la dignidad humana. Estud Const. 2013;11(2):333-68. doi: 10.4067/S071852002013000200009

7. Salas S, Arriagada A, Bernier L, Besio M, Micolich C, Misseroni A, et al. Aspectos éticos de la conducta del médico ante personas en huelga de hambre: opinión del departamento de Ética del Colegio Médico de Chile. Rev Médica Chile. 2018;146(9):1059-63. doi: 10.4067/s003498872018000901059

8. Crosby SS, Apovian CM, Grodin MA. Hunger strikes, force-feeding, and physicians' responsibilities. JAMA. 2007;298(5):563-6. doi: 10.1001/jama.298.5.563

9. WMA Declaration of Malta on Hunger Strikers [Internet]. World Medical Association; 2020 [consultado el 18 de julio de 
2021]. Disponible en: https://www.wma.net/policies-post/ wma-declaration-of-malta-on-hunger-strikers/

10. Binswanger I, Elmore JG. Clinical care of incarcerated adults [Internet]. UpToDate; 2021 [consultado el 25 de junio de 2021]. Disponible en: https://www.uptodate.com/contents/ clinical-care-of-incarcerated-adults

11. Annas GJ. Force-feeding at Guantanamo. J Med Ethics. 2017;43(1):26. doi: 10.1136/medethics-2016-103579

12. Omar M, Nouh F, Younis M, Younis M, Nouri A, Ahmed D, et al. Refeeding syndrome. SAS J Med. 2017;3(11):307-12. doi: 10.21276/sasjm.2017.3.11.4

13. Wirth R, Diekmann R, Janssen G, Fleiter O, Fricke L, Kreilkamp A, et al. Refeeding syndrome: Pathophysiology, risk factors, prevention, and treatment. Internist. 2018;59(4):32633. doi: $10.1007 /$ s00108-018-0399-0

14. Kant I. Metaphysik der sitten. Alemania: Reclam; 1990.

15. Kant I. Lecciones de ética. Barcelona: Austral; 2017.

16. Reyes-Arellano F. Perspectivas filosóficas a partir del problema del suicidio. Pers Bioét. 2021;25(1):e2514. doi: 10.5294/ pebi.2021.25.1.4

17. Miranda A. El principio del doble efecto y su relevancia en el razonamiento jurídico. Rev Chil Derecho. 2008;35(3):485519. doi: 10.4067/S0718-34372008000300005

18. Beauchamp T, Childress J. Principles of biomedical ethics. $8^{\mathrm{a}}$ edición. New York: Oxford University Press; 2019.

19. Huether G, Zhou D, Schmidt S, Wiltfang J, Rüther E. Longterm food restriction down-regulates the density of serotonin transporters in the rat frontal cortex. Biol Psychiatry. 1997;41(12):1174-80. doi: 10.1016/s0006-3223(96)00265-x
20. Robinson S, Winnik HZ. Severe psychotic disturbances following crash diet weight loss. Arch Gen Psychiatry. 1973;29(4):559-62. doi: 10.1001/archpsyc. 1973.04200040099016

21. Glucksman ML, Hirsch J. The response of obese patients to weight reduction: A clinical evaluation of behavior. Psychosom Med. 1968;30(1):1-11. doi: 10.1097/00006842196801000-00001

22. Fessler DMT. The implications of starvation induced psychological changes for the ethical treatment of hunger strikers. J Med Ethics. 2003;29(4):243-7. doi: 10.1136/jme.29.4.243

23. Rowland Jr CV. Psychotherapy of six hyperobese adults during total starvation. Arch Gen Psychiatry. 1968;18(5):541-8. doi: 10.1001/archpsyc.1968.01740050029005

24. Crumpton E, Wine DB, Drenick EJ. Starvation: stress or satisfaction? JAMA. 1966;196(5):394-6.

25. Kollar EJ, Atkinson RM. Responses of extremely obese patients to starvation. Psychosom Med. 1966;28(3):227-46. doi: 10.1097/00006842-196605000-00004

26. Beresford D. Ten men dead. The story of the 1981 Irish hunger strike. Atlantic Monthly Press; 1997.

27. Silove D, Curtis J, Mason C, Becker R. Ethical considerations in the management of asylum seekers on hunger strike. JAMA. 1996;276(5):410-5.

28. Crisci A. Tratamiento médico en contra de la voluntad del paciente. En: Andorno R, Ivone V (editores). Casos de bioética y derecho. Valencia, España: Tirant Lo Blanch; 2015. 